\title{
A Network of Three Types of Filaments Organizes Synaptic Vesicles for Storage, Mobilization, and Docking
}

\author{
Andy A. Cole, ${ }^{1,2}$ Xiaobing Chen, ${ }^{1}$ and Thomas S. Reese ${ }^{1}$ \\ ${ }^{1}$ Laboratory of Neurobiology, National Institute of Neurological Disorders and Stroke, National Institutes of Health, Bethesda, Maryland 20892, and \\ 2Department of Cellular and Molecular Biology, Feinberg School of Medicine, Northwestern University, Chicago, Illinois 60611
}

Synaptic transmission between neurons requires precise management of synaptic vesicles. While individual molecular components of the presynaptic terminal are well known, exactly how the molecules are organized into a molecular machine serving the storage and mobilization of synaptic vesicles to the active zone remains unclear. Here we report three filament types associated with synaptic vesicles in glutamatergic synapses revealed by electron microscope tomography in unstimulated, dissociated rat hippocampal neurons. One filament type, likely corresponding to the SNAREpin complex, extends from the active zone membrane and surrounds docked vesicles. A second filament type contacts all vesicles throughout the active zone and pairs vesicles together. On the third filament type, vesicles attach to side branches extending from the long filament core and form vesicle clusters that are distributed throughout the vesicle cloud and along the active zone membrane. Detailed analysis of presynaptic structure reveals how each of the three filament types interacts with synaptic vesicles, providing a means to traffic reserved and recycled vesicles from the cloud of vesicles into the docking position at the active zone.

Key words: docking; presynaptic; storage; synaptic vesicles; tomography; vesicle mobilization

\section{Significance Statement}

The formation and release of synaptic vesicles has been extensively investigated. Explanations of the release of synaptic vesicles generally begin with the movement of vesicles from the cloud into the synaptic active zone. However, the presynaptic terminal is filled with filamentous material that would appear to limit vesicular diffusion. Here, we provide a systematic description of three filament types connecting synaptic vesicles. A picture emerges illustrating how the cooperative attachment and release of these three filament types facilitate the movement of vesicles to the active zone to become docked in preparation for release.

\section{Introduction}

Brain function relies on quick synaptic communication, which requires precise control of synaptic vesicles in the presynaptic terminal. Dysfunction in the presynaptic machinery can lead to behavioral deficits (Gitler et al., 2004). A detailed list of the molecular components of the presynaptic terminal has been assembled (Takamori et al., 2006; Imig et al., 2014), but the picture of

\footnotetext{
Received Aug. 4, 2015; revised Jan. 29, 2016; accepted Feb. 5, 2016.

Author contributions: A.A.C. designed research; A.A.C. and X.C. performed research; A.A.C., X.C., and T.S.R. analyzed data; A.A.C. and T.S.R. wrote the paper.

This research was supported by the Intramural Research Program of the National Institutes of Health (NIH), National Institute of Neurological Disorders and Stroke. We thank Dr. Richard Leapman, National Institute of Biomedical Imaging and Bioengineering, $\mathrm{NHH}$, for the use of his FEI Tecnai $300 \mathrm{kV}$ electron microscope and his support in the acquisition of tomographic data; Rita Azzam and Virginia Crocker for preparing the sections for tomography; and Christine Winters for preparing hippocampal cultures, as well as performing high-pressure freezing and freezesubstitution for tomography.

Correspondence should be addressed to Andy A. Cole, Laboratory of Neurobiology, National Institute of Neurological Disorders and Stroke, National Institutes of Health, 49 Convent Drive, Room 3A68, Bethesda, MD 20892. E-mail: cole.andy.a@gmail.com.

DOI:10.1523/JNEUROSCI.2939-15.2016

Copyright $\odot 2016$ the authors $\quad 0270-6474 / 16 / 363222-09 \$ 15.00 / 0$
}

how presynaptic molecular components are organized into a molecular machine able to rapidly mobilize and release vesicles is far from complete.

To a large extent, the lack of structural information about the organization of presynaptic machinery is a result of the labile nature of structures in the presynaptic terminal. Fortunately, freezing on the order of milliseconds preserves cytoplasmic structures with little distortion or unwanted stimulation (Moore, 1987; Dubochet, 1995). In fact, freeze-etching of slam-frozen brain exposes filamentous material associated with vesicles near the active zone (AZ) in remarkable detail (Landis et al., 1988). However, electron micrographs of freeze-etching only provide a clear view of structures at the replica surface, and yield only a glance at the larger organization of filaments in the vesicle cloud (Landis et al., 1988; Hirokawa et al., 1989).

The introduction of high-pressure freezing in conjunction with electron microscope (EM) tomography now provides access to structures much deeper in the specimen, making it possible to visualize clearly presynaptic filamentous material and presynaptic machinery (Siksou et al., 2011). EM tomography combined 
Table 1. Enumeration of active zone and synaptic filaments, listed by experiment

\begin{tabular}{lccc}
\hline & Experiment 1 & Experiment 2 & Both \\
\hline Virtual section thickness $(\mathrm{nm})$ & 1.4 & 1.4 & 1.4 \\
Active zone area sampled $\left(\mathrm{nm}^{2}\right)$ & 29,000 & 39,000 & 68,000 \\
Presynaptic volume sampled $\left(\mathrm{nm}^{3}\right)$ & $7.25 \times 10^{6}$ & $9.75 \times 10^{6}$ & $17 \times 10^{6}$ \\
All vesicles & 89 & 113 & 202 \\
All filaments & 237 & 245 & 482 \\
Docking filaments & 31 & 39 & 70 \\
Bridge filaments & 147 & 154 & 301 \\
Cluster filaments & 59 & 52 & 111 \\
\hline
\end{tabular}

with high-pressure freezing and freeze-substitution can produce high-resolution three-dimensional (3D) reconstructions of filament organization deep within the synapse (Chen et al., 2008b) and is particularly well suited to visualize the entirety of the filamentous network within the vesicle cloud. Using EM tomography on high-pressure frozen, freeze-substituted 3-week-old cultures of dissociated rat hippocampal neurons, we produced three-dimensional renderings of all filaments associated with vesicles in the synaptic vesicle cloud within $250 \mathrm{~nm}$ of the active zone, revealing their three-dimensional organization.
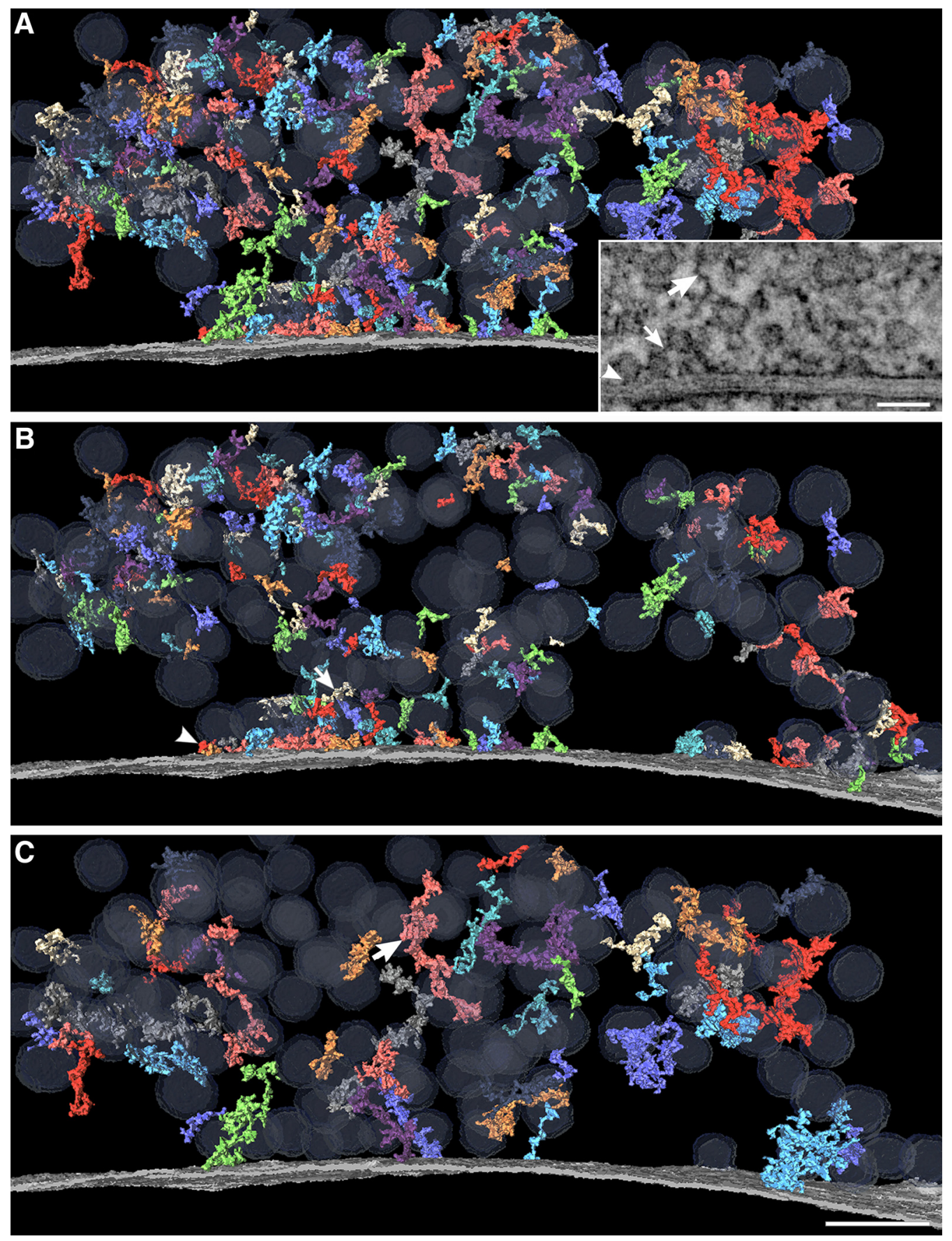

Figure 1. EM tomography of hippocampal synapses reveals filaments connecting synaptic vesicles to the membrane and each other throughout the vesicle cloud. $A$, Rendering of all membrane to membrane filament types within $250 \mathrm{~nm}$ of the AZ. The inset features a 30-nm-thick projection image of the synapse with electron-dense material that corresponds to the docking (arrowhead), bridge (small arrow), and cluster (large arrow) filaments indicated. Filaments are arbitrarily colored for contrast, while synaptic membrane and vesicles are gray and transparent gray, respectively. $B$, Rendering of docking filaments from the AZ (arrowhead) and bridge filaments within the vesicle cloud (small arrow). C, Rendering of long, modular cluster filaments to the AZ and in the cloud (large arrow). Scale bars, $50 \mathrm{~nm}$. 

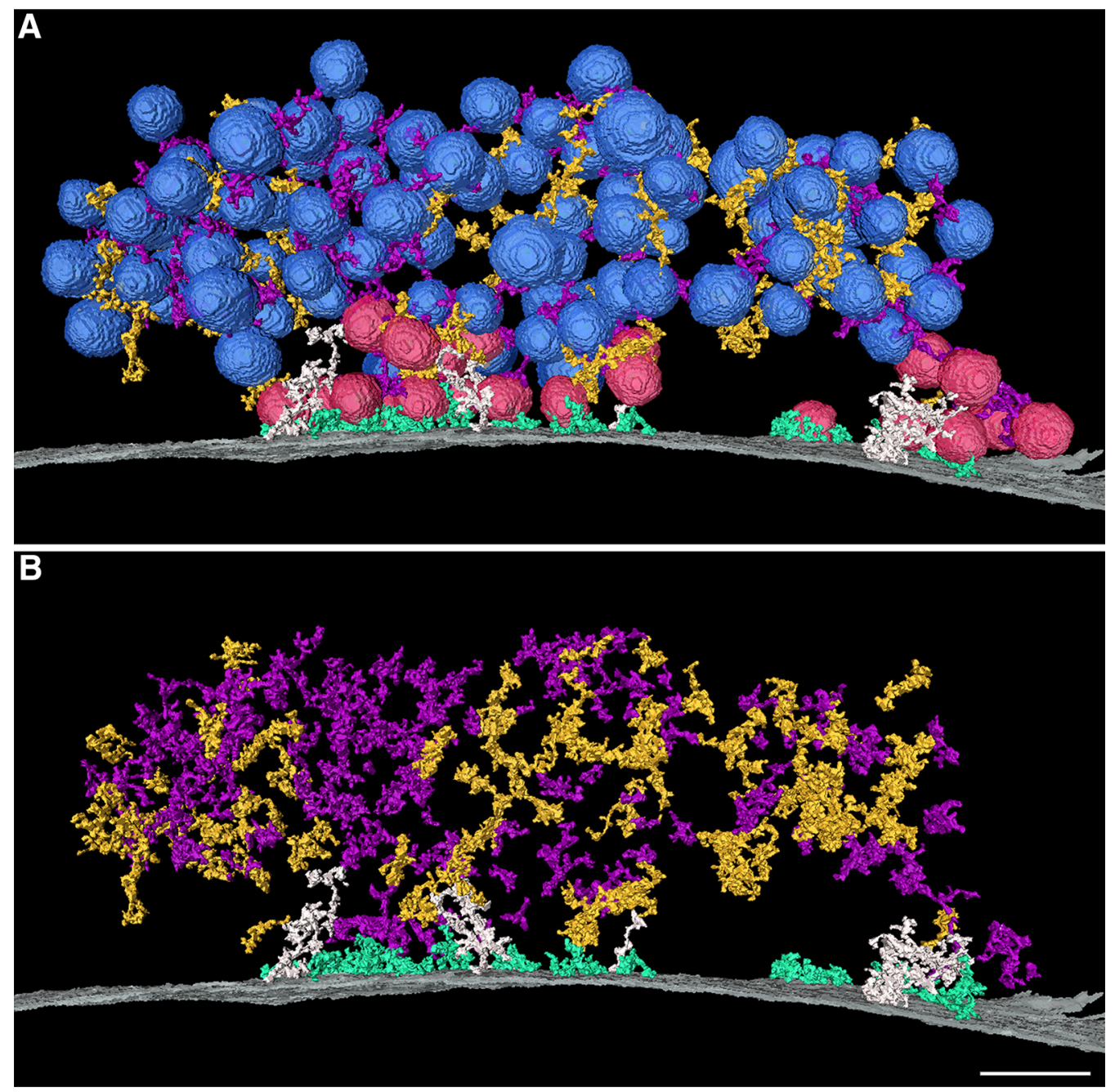

Figure 2. Three types of filaments connect to synaptic vesicles. A, All synaptic vesicles (blue, or red if directly connected to filaments extending from the active zone membrane) link to the active zone membrane or each other by filamentous material, forming a coherent group of vesicles termed a vesicle cloud. Typically, four small docking filaments (teal) from the active zone surround each vesicle that contacts the active zone. Longer cluster filaments are found either extending from the active zone (white) or free throughout the vesicle cloud (gold). Small bridge filaments (purple) are distributed throughout the cloud and pair vesicles. $\boldsymbol{B}$, Same view and color code as in $\boldsymbol{A}$, but vesicles are removed to expose the underlying filamentous network. Scale bar, $50 \mathrm{~nm}$.

The three-dimensional reconstructions reveal that three filament types dominate the cytoplasmic structure of these presynaptic terminals. The renderings provide new structural details on the shapes and connections of each filament type and show that each filament associates with synaptic vesicles. Many vesicles connect to all three filament types. The complex connectivity of vesicles with the different filament types produces an organized network extending out from the active zone. We propose that the three filament types form an interlocking system that provides the means to traffic vesicles all the way to fusion-competent areas of the synaptic membrane from deeper regions of the vesicle cloud.

\section{Materials and Methods}

Cultures. Dissociated rat hippocampal neurons of either sex (embryonic day 20) were plated on a glial cell feeder layer in Bal-Tec $3 \mathrm{~mm}$ gold specimen chambers and incubated for 3 weeks in $10 \% \mathrm{CO}_{2}$ at $35^{\circ} \mathrm{C}$ in a medium consisting of MEM with $6 \mathrm{~g} / \mathrm{L}$ glucose (320-330 Osm), $2 \mathrm{~mm}$ Glutamax 1, 2\% fetal bovine serum, 5\% horse serum, and N3 (a growth factor cocktail of apotransferrin, putrescine, selenium, triiodothyronine, insulin, progesterone, and corticosterone; Mayer and Vyklicky, 1989; Chen et al., 2011).

High-pressure freezing and freeze-substitution. Gold specimen chambers, with cultured neurons, were immersed in $124 \mathrm{~mm} \mathrm{NaCl}, 2 \mathrm{mM} \mathrm{KCl}$,
$1.24 \mathrm{~mm} \mathrm{KH}_{2} \mathrm{PO}_{4}, 1.3 \mathrm{~mm} \mathrm{MgCl}_{2}, 2.5 \mathrm{~mm} \mathrm{CaCl}_{2}, 30 \mathrm{~mm}$ glucose, $25 \mathrm{~mm}$ HEPES, and $0.5 \%$ ovalbumin, pH 7.4 (325 Osm). Hexadecane was applied directly to the top of the specimen chamber immediately before specimen freezing, as a space filler, to eliminate air bubbles. Specimens were frozen at $\sim 2100$ bar in a BAL-TEC HPM 010 (TECHNOTRADE International).

Saturated uranyl acetate in HPLC-grade acetone was frozen in liquid nitrogen in a glass scintillation vial. Four percent acrolein in acetone was layered on top of the uranyl acetate and immersed in liquid nitrogen. The vial was placed in an AFS Unit (Leica Microsystems). The frozen specimens were transferred into the prepared glass vials for freeze-substitution.

The AFS Unit (Leica Microsystems) was programmed to execute the following schedule: hold at $-160^{\circ} \mathrm{C}$ for $15 \mathrm{~min}$; rise from -160 to $-90^{\circ} \mathrm{C}$ over $14 \mathrm{~h}$; hold at $-90^{\circ} \mathrm{C}$ for $8 \mathrm{~h}$; rise from -90 to $-60^{\circ} \mathrm{C}$ over $6 \mathrm{~h}$; and hold at $-60^{\circ} \mathrm{C}$ for $12 \mathrm{~h}$. Samples were put in Lowicryl HM20 resin in acetone and polymerized in the AFS Unit (Leica Microsystems) at $-50^{\circ} \mathrm{C}$ over $2 \mathrm{~d}$; then, the temperature was raised to $20^{\circ} \mathrm{C}$ over $70 \mathrm{~h}$ (Chen et al., 2014). Thin sections $100-200 \mathrm{~nm}$ thick, cut en face, were mounted on carbon-coated formvar grids. Fiducial markers (10 nm gold particles) were added to both sides of the grid as fiducial markers for calculating tomographic reconstructions (Chen et al., 2008b).

EM tomography. Spine synapses were selected and imaged on an FEI Tecnai $300 \mathrm{kV}$ electron microscope with a field-emission gun. Each syn- 
apse was imaged in two orthogonal axes of tilt at $2^{\circ}$ increments from $74^{\circ}$ to $-74^{\circ}$. Pixel size was either 0.48 or $0.75 \mathrm{~nm}$ in a $2048 \times 2048$ image, but was double binned, making for a final pixel size of 1 or $1.4 \mathrm{~nm}$ in the final $3 \mathrm{D}$ volume. The image series from separate tilt axis sets were reconstructed individually and merged in IMOD software (Kremer et al., 1996) with an estimated average alignment error of $<0.3$ pixels. The resulting 3 D volume was analyzed with Amira software (FEI Visualization Sciences Group; Chen et al., 2008a). Projection images from tomograms are made by averaging six consecutive 1.4-nm-thick virtual sections together in Amira.

Segmentation. Each 3D volume was divided into 1-voxel-thick virtual sections. Segmentation consisted of tracing an object from one section to the next, because semiautomatic selection techniques in Amira, such as thresholding, led to convoluted renderings. Manual segmentation of structures in three orthogonal planes, with specific criteria (examples given below), led to complete and interpretable renderings. An object was segmented and classified as a synaptic vesicle if it was approximately spherical, had a single lipid bilayer membrane, and was between 20 and $60 \mathrm{~nm}$ in diameter. The center line of the single bilayer membrane was segmented in three orthogonal planes to produce a continuous sphere. The synaptic active zone membrane was similarly segmented to produce a membrane sheet. An object was segmented and classified as a presynaptic filament if it continuously spanned at least two synaptic vesicles or a single vesicle to the active zone membrane. The segmentation of a filamentous object ended at a point where it became discontinuous or when a drastic change in morphology occurred near a vesicle or active zone membrane.

Rendering and measurements. Surface-rendering algorithms trim and smooth segmented materials to generate rendered surfaces. This can make some small filaments appear shorter or discontinuous. The Amira surface-rendering algorithm was used to produce renderings shown here without additional smoothing. Segmentation was not bloated to compensate for surface-rendering alterations. All measurements were made using the Amira 3D measurement tool on surface renderings, unless a full, undistorted cross-sectional view existed in a virtual section, allowing an object to be measured directly on the data. The longest continuous path along the filament between membrane contact points was considered the filament length.

\section{Results}

Tomograms were made of resting asymmetric spine synapses (neither stimulated nor inhibited) from dissociated hippocampal cultures, with the aim of analyzing synaptic proteins. EM tomography of freeze-substituted cultures provided more detailed images of the filaments than were available with standard thin-section TEM. This improvement made possible the individual segmentation of filaments and vesicles, in 1.4-nm-thick virtual sections. The tomograms were segmented and rendered from specimens prepared earlier (Chen et al., 2008a). Complete renderings of filament contacts with vesicles extending up to 250 $\mathrm{nm}$ from the AZ were derived from two tomograms (Table 1). Active zones appeared as dense areas adjacent to postsynaptic densities in dendritic spines. Three types of filaments contacted synaptic vesicles, linking them to each other and the active zone membrane to produce a coherent cloud (Fig. 1). Filament contacts will be referred to as "connections" hereafter, on the assumption that the filaments are attached, however temporarily, to the vesicles or active zone membrane. All synaptic vesicles were connected by filaments.

\section{Filament types}

Three types of filaments contacting synaptic vesicles could be distinguished primarily by their shapes and lengths. One type averaged $22 \mathrm{~nm}$ long and was straight, manifesting a globular base where it contacts the AZ membrane. A second type was also straight but lacked a globular base and was often some-
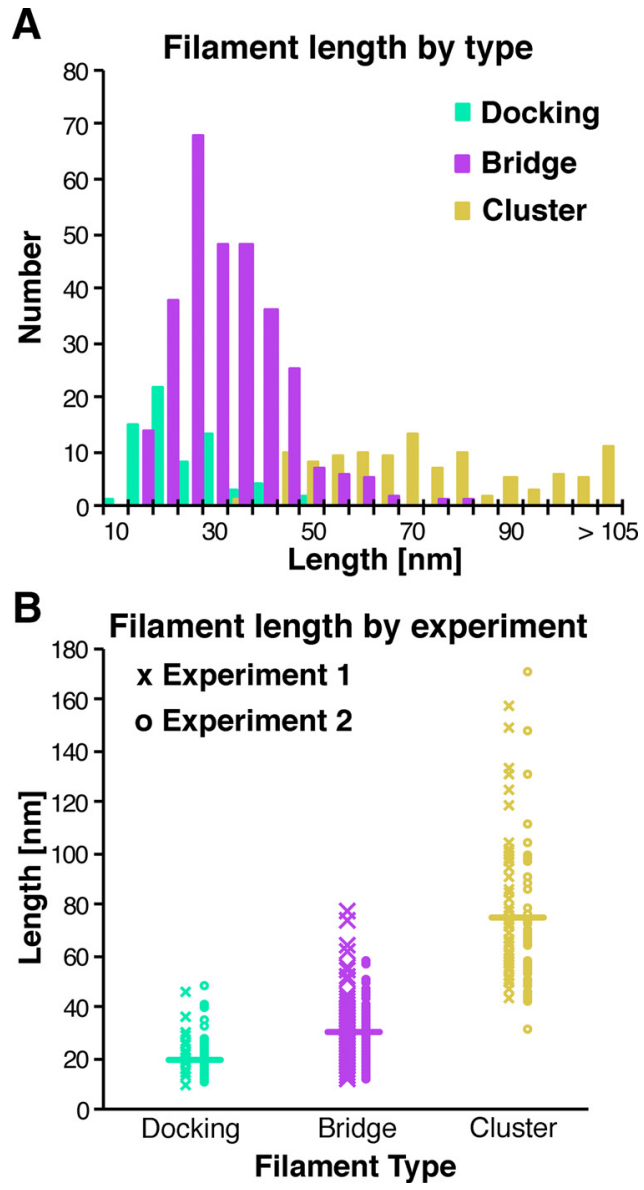

Figure 3. Distributions of lengths of three types of synaptic filament. $A$, Histogram of lengths of 70 docking filaments, 301 bridge filaments, and 111 cluster filaments pooled from two experiments. $\boldsymbol{B}$, Scattergrams of the lengths of the same three types of filaments showing that their distributions in the two experiments are indistinguishable. Crossbars indicate the combined filament type mean. Cluster filaments are the most variable in length.

Table 2. Dimensions and properties of synaptic filaments by type

\begin{tabular}{lccc}
\hline & Docking & Bridge & Cluster \\
\hline Filament population & $15 \%^{a}$ & $62 \%^{a}$ & $23 \%^{a}$ \\
Contact the AZ & $100 \%^{b}$ & $0 \%^{b}$ & $15 \%^{b}$ \\
Number of vesicle contacts (range) & $1(1-2)$ & $2(2-4)$ & $3(1-8)$ \\
Filament length (range) (nm) & $22(10-47)$ & $30(11-78)$ & $74(30-169)$ \\
Filament diameter (range) (nm) & $5(3-8)$ & $8(5-16)$ & $5(2-9)$ \\
\hline
\end{tabular}

${ }^{a}$ Percentage of all filaments sampled.

${ }^{b}$ Percentage of all filaments of a type sampled.

what longer. A third type of filament was much longer $(\sim 70$ $\mathrm{nm}$ ), not straight, and was distinguished by kinks and branches along its length (Figs. 2, 3, Table 2).

The straight filaments with globular bases were 10 and $47 \mathrm{~nm}$ long, with a mean length of $22 \mathrm{~nm}$ (Figs. 4, 5). The stalks of these filaments averaged $5 \mathrm{~nm}$ in diameter, but they could thicken to more than twice that diameter at their globular base. This type of filament exclusively connects vesicles to the AZ membrane (Table 2), and accounted for $15 \%$ of the filaments in the sampled volume. Each "docking filament" contacted one synaptic vesicle and could assume any orientation, from vertical to horizontal, with respect to the AZ membrane. Some filaments lay almost parallel to the $A Z$ membrane when the vesicle it connected was very close to the $\mathrm{AZ}$ membrane. 
The second type of straight filament, which lacked a globular base, was 11-78 $\mathrm{nm}$ long with a mean length of $30 \mathrm{~nm}$. These filaments were distinctly thicker and more variable in midsection diameter than docking filaments. Filaments of this type of were tilted between 0 and $45^{\circ}$ with respect to the $\mathrm{AZ}$ plane. They made up $62 \%$ of the total population of filaments in the volume sampled and made bridges between vesicles throughout the cloud. These "bridge filaments" typically joined vesicles into pairs (Fig. 6D-F, Table 2), but some bridge filaments contacted the surface of one vesicle while spanning between two other vesicles.

The third type of filament was 30-169 $\mathrm{nm}$ long and manifested distinct kinks along its axis at intervals of $\sim 13 \mathrm{~nm}$, from which side branches might extend. Its central stalk was $\sim 5 \mathrm{~nm}$ in diameter. Side branches originating from kinks were 22 $\mathrm{nm}$ long and $4 \mathrm{~nm}$ in diameter (Fig. 6B), and typically contacted a synaptic vesicle, organizing them into small clusters along the central stalk. As many as eight synaptic vesicles were connected to one "cluster filament". Cluster filaments assumed a variety of orientations, but, unlike bridge filaments, they could be oriented at $90^{\circ}$ with respect to the AZ membrane. Cluster filaments were distributed throughout the vesicle cloud and accounted for $23 \%$ of the filaments in the volume sampled. Fifteen percent of cluster filaments contacted the AZ membrane (Fig. 5). Cluster filaments contacting the AZ were 47-102 $\mathrm{nm}$ long, which is somewhat shorter than those cluster filaments not contacting the AZ. Cluster filaments contacting the AZ also oriented with a steep horizontal lean with respect to the $\mathrm{AZ}$ membrane. The kinks in their stalks distinguished the shorter cluster filaments from longer bridge filaments.

\section{Filaments connected to the $\mathrm{AZ}$ membrane}

The AZ was replete with filament connections. Both docking and cluster filament types made connections to the AZ membrane (Table 2). Docking filaments outnumbered cluster filaments by a ratio of 3 to 1 . Docking filaments associated with vesicles within $16 \mathrm{~nm}$ of the $\mathrm{AZ}$ and surrounded the bases of vesicles in direct contact with the AZ, typically in groups of two to six (mean, four; Fig. 4). Docking filaments appeared to be oriented horizontal more often when a larger portion of the vesicle surface area contacted the AZ. A single vesicle was found fused with the AZ membrane, and electron-dense material surrounded the connection between vesicle and AZ membrane. This material was bulkier than the typical docking filament type, and it lay along the membrane.

Docking filaments connected with vesicles that made contact with or lay near the AZ membrane. However, vesicles not contacting the AZ membrane made only a single docking filament
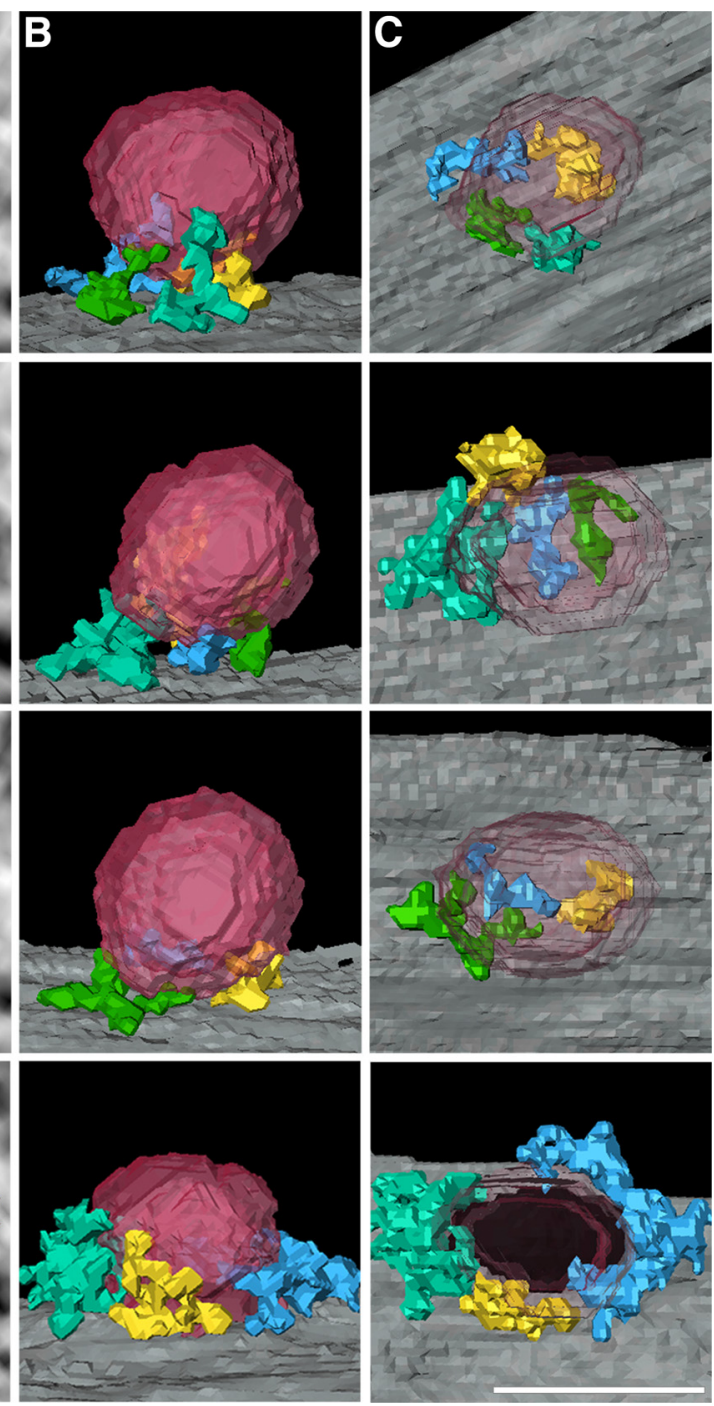

Figure 4. Small filaments extend from the active zone membrane to surround docked vesicles. $\boldsymbol{A}$ features projection images of synaptic vesicle (red) to which they connect. $\boldsymbol{C}$ shows the rendering in $\boldsymbol{B}$ from an en face view. The bottom panels of $\boldsymbol{A}-\boldsymbol{C}$ feature a fused vesicle. Scale bars, $35 \mathrm{~nm}$.

connection, if any at all. In contrast, cluster filaments at the AZ connected any vesicles in reach near the AZ, whether or not a vesicle contacted the active zone (Fig. 5). These cluster filaments were between 47 and $102 \mathrm{~nm}$ in length but often assumed a horizontal lean that limited their vertical reach to $\sim 50 \mathrm{~nm}$. Though limited in reach, cluster filaments on the AZ membrane linked the $\mathrm{AZ}$ to the vesicle cloud.

\section{Filament network}

Enumeration of filament connections was crucial to understanding the patterns underlying vesicle organization (see Discussion). Docking and cluster filaments both connect the vesicle cloud to the AZ membrane (Fig. 2, Table 3). Vesicles directly connected to the $\mathrm{AZ}$ were still integrated into the vesicle cloud by bridge and cluster filaments (Fig. 7). A single vesicle fused with the AZ membrane was not integrated into the vesicle cloud.

No barrier separated vesicles connected to the AZ from other vesicles. In fact, all vesicles, regardless of their physical location, made connections with cluster filaments, bridge filaments, or 


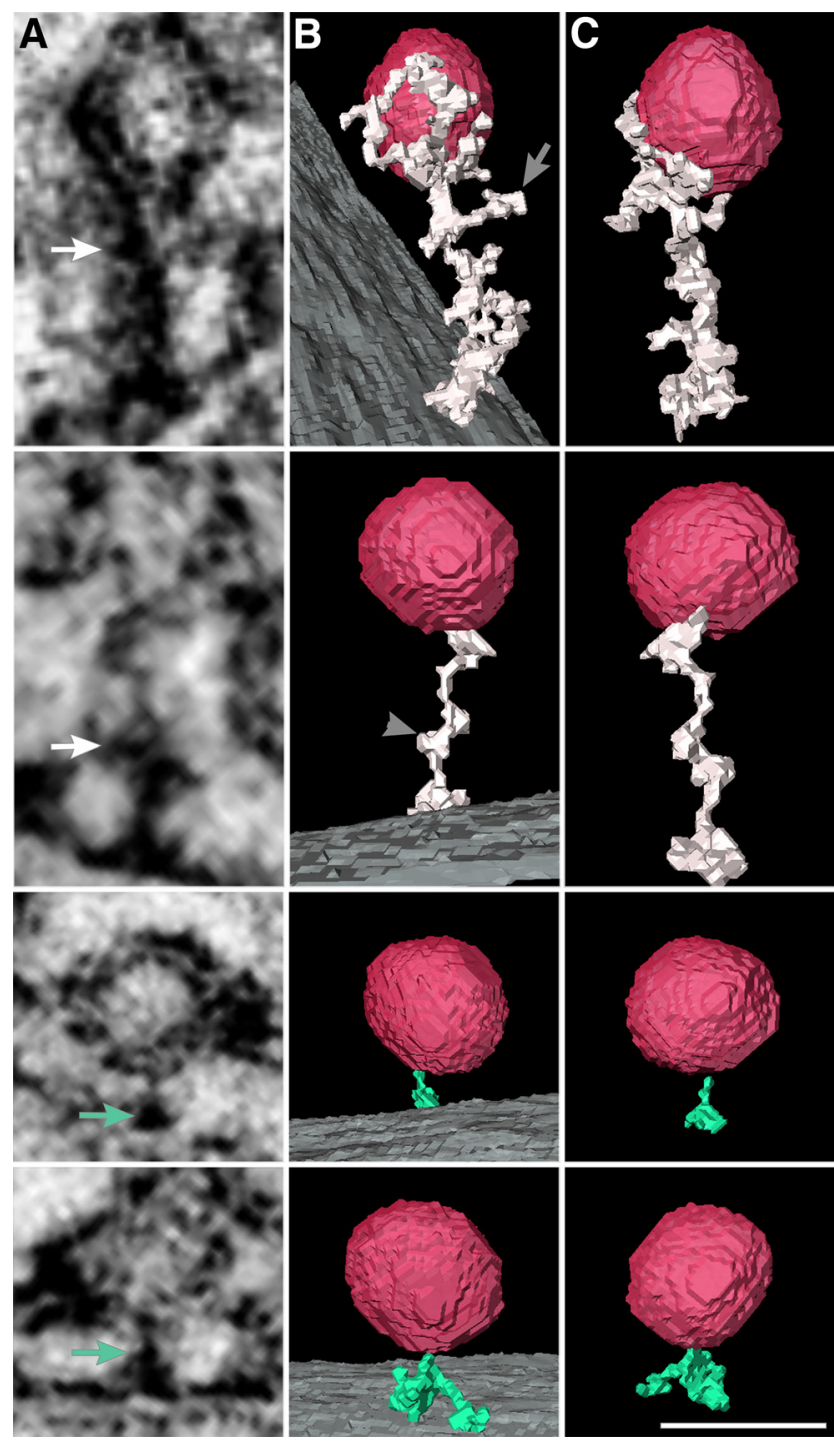

Figure 5. Two filament types associate with cloud vesicles and the active zone membrane. $A, B$, and $C$ show three different views of four vesicles (red) that do not contact the active zone but have filament connections to it. $A$ features projection images of each vesicle. Colored arrows indicate electron-dense material that correspond to filaments rendered in the same color in $\boldsymbol{B}$ and $\boldsymbol{C}$. $\boldsymbol{B}$ features the three-dimensional renderings of long, modular filamentous cluster filaments (white) or singular docking filaments (teal). Both filament types are shown extending from the active zone. C shows the rendering in $\boldsymbol{B}$ from another view. The kinks (gray arrowhead) that are characteristic of a cluster filament are often accompanied by a side branch (gray arrow). Scale bar, $35 \mathrm{~nm}$.

both (Table 3 ). Only 6 of 202 vesicles did not connect with a bridge filament. The median connectivity of a single vesicle was three bridge and one cluster filament connection. A total of 79\% of vesicles associated with both cluster and bridge filaments. The number of cluster filament connections increased with the distance from the active zone, and synaptic vesicles can connect to more than one cluster filament. These multiple connections link cluster filaments together, and when three or more cluster filaments are linked in series, we refer to the larger cluster of vesicles as a supercluster. The vesicle cloud was less dense further away from superclusters. There were two and three superclusters in Experiment 1 and 2, respectively. Four of five of the superclusters extended deep into the vesicle cloud from the active zone membrane. Only $18 \%$ of vesicles are not connected to cluster filaments, and these vesicles lay along the edges of superclusters
(Fig. 7). Unidentified presynaptic structures occupied areas along the active zone membrane but were not rendered.

\section{Discussion}

EM tomography combined with freeze-substitution represents an advance toward imaging filaments in presynaptic terminals at a level of detail previously offered by the freeze-etch technique (Landis et al., 1988; Frank et al., 1995; Baumeister et al., 1999; Harlow et al., 2001; McIntosh, 2001; Zampighi et al., 2006; Fernández-Busnadiego et al., 2010; Burette et al., 2012). We take advantage of the three-dimensional reconstructions provided by EM tomography and freeze-substitution to analyze the system of filaments connecting vesicles in brain synapses. Evidence that dynamic protein organization, which is best studied in the absence of cross-linking fixatives (Smith and Reese, 1980), has constrained work so far to disassociated hippocampal cultures grown with glial cells (Chen et al., 2008a). Synapses in the dissociated hippocampal cultures differed in some respects from those in the hippocampus of intact brain, in that there are far more autapses and few or no mushroom spines in the cultures. However, direct comparison of the structural and physiological properties of synapses in hippocampal cultures and corresponding synapses in intact brain suggests that they are generally similar (Schikorski and Stevens, 1997).

\section{Three distinct types of filaments contact synaptic vesicles} Every vesicle in the vesicle cloud surrounding the active zone is integrated into an interlocking network of docking, bridge, and cluster filaments. Though synaptic vesicles contacting the active zone membrane are generally considered to be docked (Stigloher et al., 2011; Watanabe et al., 2013; Imig et al., 2014), EM tomography now shows that docked vesicles typically have three or more docking filament connections around their base, similar to pins on docked vesicles in the neuromuscular junction (Szule et al., 2012). However, pins ( $\sim 9 \mathrm{~nm})$ are shorter than docking filaments ( $\sim 22 \mathrm{~nm}$; Szule et al., 2012). Some docking filaments are oriented horizontally, while others are vertical to the membrane, indicating variations between docked vesicles. If horizontal orientation of all the docking filaments between a vesicle and the presynaptic membrane is indicative of fusion-competent primed vesicles, the low frequency of this type of configuration might reflect the low probability of the release of vesicles in hippocampal cultures (Allen and Stevens, 1994; Stevens and Wang, 1994; Murthy, 1997). Docking filaments also associate with synaptic vesicles up to $\sim 15 \mathrm{~nm}$ from the active zone, but each of these vesicles connect to only one docking filament. The variations in docking filament orientation and connectivity are depicted in Figure 8 .

At both synapses examined, $97 \%$ of all vesicles connected to bridge filaments. A vesicle can contact up to 12 bridge filaments, as reported for short presynaptic connector filaments (Gustafsson et al., 2002; Burette et al., 2012; Szule et al., 2012). Bridge filaments show small variations in morphology, indicating that they may not be entirely homogeneous.

A third filament type, cluster filaments, are similar in length $(30-169 \mathrm{~nm})$ to filaments previously reported to extend outward only from active zones (Landis et al., 1988; Gotow et al., 1991; Siksou et al., 2007, 2009; Burette et al., 2012). However, cluster filaments are shown here to be distributed throughout the presynaptic terminal and to possess periodic kinks with side branches along their long axis by which they cluster many vesicles. Approximately $80 \%$ of vesicles connect to at least one cluster filament. Though not characterized, filaments resembling cluster 
filaments are apparent in the cloud and at the active zone of other rendered images of presynaptic filaments (Stigloher et al., 2011).

Synaptic vesicles crowd together and fill the area surrounding the active zone (Siksou et al., 2007; Fernández-Busnadiego et al., 2010; Burette et al., 2012). Cluster filaments can be wedged within crowds of vesicles such that a complete picture of their connections is obscured. Indeed, vesicles are usually connected by both cluster and bridge-type filaments. Vesicles can connect to more than one cluster filament, linking vesicle clusters together. Three or more vesicle clusters chained together form a supercluster (Fig. 8). The density of vesicles with multiple cluster filament connections increases in more distal regions, resulting in a tight cluster filament mesh that likely limits the mobility of vesicles. Vesicles without a cluster filament connection have bridge filament connections, and, in this way, vesicles connect across gaps between superclusters to form a continuous cloud.

\section{Identities of filaments}

Details of size, shape, and distribution may permit tentative identification of filaments. Vesicles connected by multiple filaments to the active zone are considered most likely to be released upon stimulation (Fernández-Busnadiego et al., 2010; Watanabe et al., 2013). As docking filaments are the only short filament type that surrounds docked vesicles, they likely correspond to a molecule or molecular complex for docking and readying vesicles for release, and may correspond to SNAREpin complexes. Like docking filaments, multiple SNAREpins surround docked vesicles (Rickman et al., 2005), and, like docking filaments, SNAREpins orient both vertically (trans-SNARE complex) and horizontally (cis-SNARE complex; Südhof and Rothman, 2009). SNAREpin complexes begin assembling vertically at $\sim 20 \mathrm{~nm}$ from the active zone membrane, but SNAREpin assemblies will zipper up to within 2 and 4 $\mathrm{nm}$ of the active zone membrane (Li et al., 2007). Thus, we suggest that docking filaments correspond to SNAREpin complexes.

Bridge filaments resemble the structure reported for synapsins coupling vesicles (Landis et al., 1988; Hirokawa et al., 1989). Knockout of synapsins markedly diminishes the numbers of filaments between synaptic vesicles (Gitler et al., 2004; Siksou et al., 2007), suggesting that synapsins constitute the majority of bridge filaments. On the other hand, the variability in bridge filament structure indicates that bridge filaments may include additional molecular components. The wide variation in the lengths of bridge and docking filaments remains unexplained.

Cluster filaments kink at regular points along their length, and side branches often extend from the kinks, so filament length depends on the number of kinks. Thus, a cluster filament appears as a complex composed of self-associated molecules with side branches. These features have yet to be matched with known molecules, but synaptic vesicles have numerous proteins on their
Table 3. Enumeration of vesicle connections to filament types, listed by experiment

\begin{tabular}{lccc}
\hline & Experiment 1 & Experiment 2 & Both \\
\hline Synaptic vesicles & 89 & 113 & 202 \\
Docked vesicles & 5 & 8 & $6 \%^{a}$ \\
Docked vesicles to docking filaments & 5 & 8 & $100 \%^{b}$ \\
Docked vesicles to bridge filaments & 5 & 6 & $85 \%^{b}$ \\
Docked vesicles to cluster filaments & 2 & 9 & $85 \%^{b}$ \\
Vesicles to docking filaments & 14 & 13 & $13 \%^{a}$ \\
Vesicles to bridge filaments & 87 & 109 & $97 \%^{a}$ \\
Vesicles to cluster filaments & 81 & 85 & $82 \%^{a}$ \\
Vesicles to both cluster and bridge filaments & 79 & 81 & $79 \%^{a}$ \\
\hline
\end{tabular}

${ }^{a}$ Percentage of all vesicles sampled.

${ }^{b}$ Percentage of all docked vesicles sampled.

surfaces, some extending into the surrounding cytoplasm where they could participate in the side arms of cluster filaments (Takamori et al., 2006).

\section{Roles of three types of filaments}

Every vesicle is integrated into one network of interlocking filaments, regardless of position in the cloud, typically by three or more filaments. Active zone cluster filaments and docked vesicles 

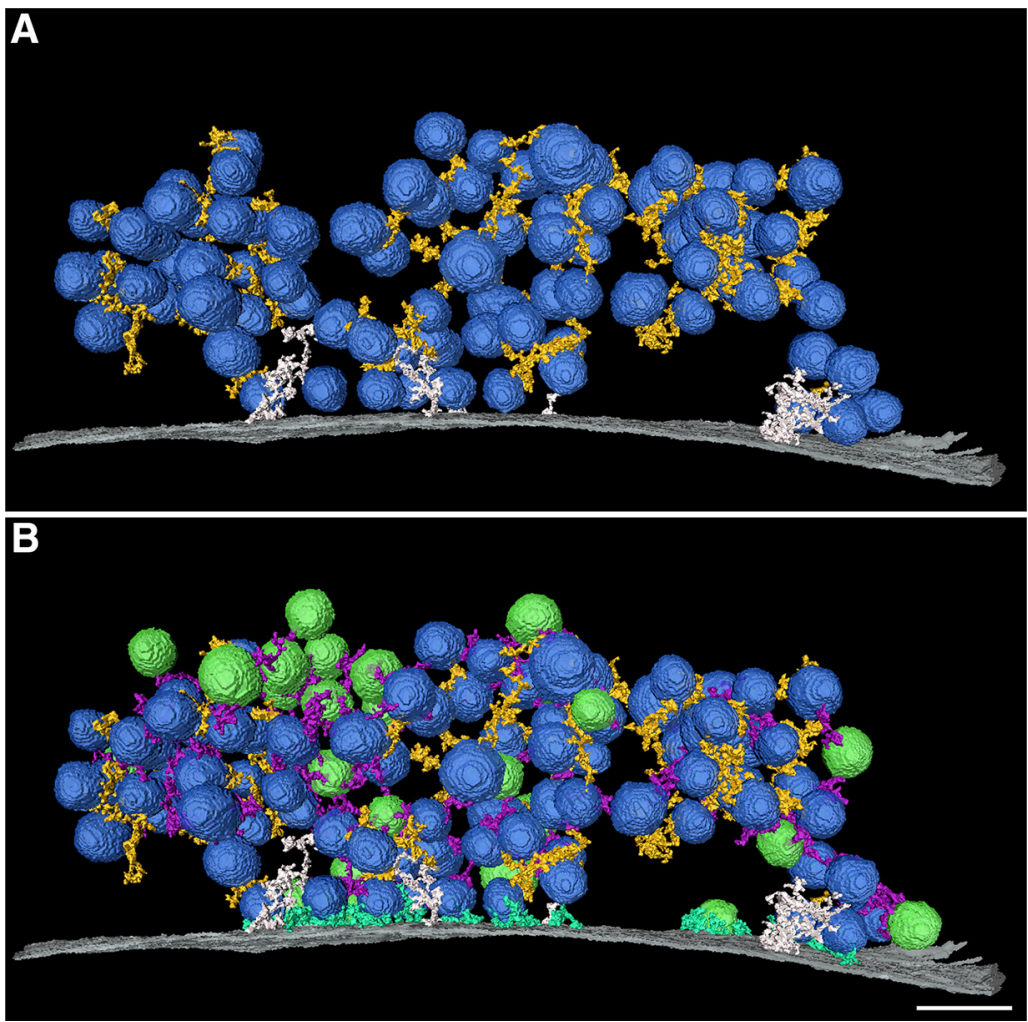

Figure 7. Bridge filament connections and superclusters link all synaptic vesicles into a single cloud. $\boldsymbol{A}$, Within the first $250 \mathrm{~nm}$ from the active zone, all cluster filaments are shown (in gold, or white if they touch the active zone) with the vesicles they connect to (blue). Vesicles can connect to multiple cluster filaments, which produce large knots of vesicles that are referred to as superclusters. $\boldsymbol{B}$, Bridging filaments (purple) and docking filaments (teal) are added to the rendering in $\boldsymbol{A}$ as well as synaptic vesicles (green) that only connect with bridge or docking filaments. Scale bar, $50 \mathrm{~nm}$.
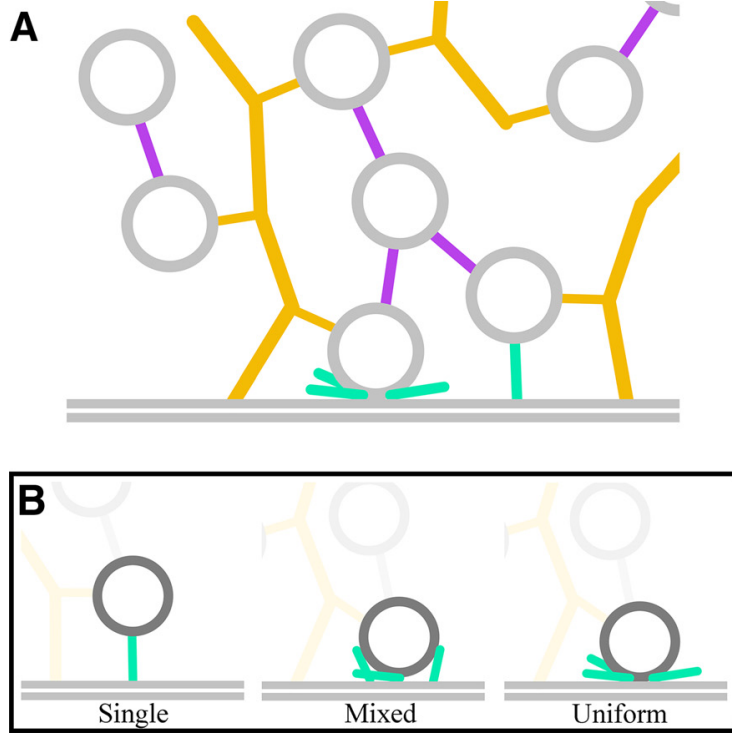

Figure 8. Diagram of the presynaptic filamentous network. $A$, Patterns of filamentous connectivity in the vicinity of the active zone are shown in their simplest form. Bridge filaments (purple) pair with vesicles (gray), while cluster filaments (gold) hold many vesicles on side branches. Docking filaments (teal) extend from the active zone membrane (parallel gray lines) to contact nearby vesicles. $\boldsymbol{B}$, Docking filament configurations. The transition between a single docking filament to a presumed fusion-competent, uniformly horizontal docking filament orientation could exert a downward force on any attached filament connections. C, A simplified linkage of cluster filaments by vesicles with more than one cluster filament connection. Tiers of vertically oriented cluster filaments form chains of vesicles extending deep into the vesicle cloud. We hypothesize that bridge filaments maintain the cohesiveness of the vesicle cloud and inhibit vesicle docking. During activity, bridge filaments likely detach from vesicles, while cluster filament connections are maintained. In this way, the collapse of a vesicle during fusion would exert a downward force on cluster filament side branches (see Discussion). anchor this network to the active zone membrane. Under resting conditions, the diffusion of vesicles should be quite limited. However, upon intense stimulation, vesicle clusters may break up into columns that attached to active zones but extended deep into the synapse (Heuser et al., 1971). Thus, cluster filament connections appear to be more stable than other filament connections. The dense cluster filament interconnection of vesicles deep in the cloud, which likely corresponds to what is commonly referred to as the "resting pool of vesicles" (Fowler and Staras, 2015), must limit the ability of vesicles to move toward the active zone.

Physiological levels of stimulation are unlikely to lead to the global changes in the connections between vesicles that have been reported after intense stimulation (Denker et al., 2011). Thus, physiological conditions may be more closely represented by the tightly clustered cloud of interconnected vesicles we show here in synapses that are neither silenced nor stimulated. At low levels of stimulation, the majority of vesicles should remain enmeshed in a network of docking, bridge, and cluster filaments. Mobilization schemes that depend on the diffusion of vesicles are likely not to be generally applicable.

In the situation mentioned above, a relaxation of local vesicle connections to bridge or cluster filaments would have to precede vesicle movements into the active zone. In fact, enzymatic loosening of synapsin connections, caused by a local increase of calcium concentration, is required for the release of synaptic vesicles (Llinás et al., 1985; Chi et al., 2001). A local loosening of the bridge filament (synapsin) network may allow the movement of vesicles to the active zone. However, this notion leaves open the question of what force moves vesicles to the active zone to replace vesicles lost from exocytosis of primed vesicles. FernándezBusnadiego et al. (2010) and Szule et al. (2012) conclude that presynaptic filaments must have some role in the movement of vesicles to the active zone, but Siksou et al. (2011) point out that a "guide" is lacking to lead vesicles to the active zone sites. Cluster filaments provide a possible way to explain vesicle mobilization. Docking filaments provide a direct connection to the active zone membrane, where vesicles show a range of docking filament configurations. We suggest that a complement of horizontal docking filaments indicates a primed vesicle, if docking filaments correspond to SNAREpin complexes, as previously pro- 
posed. The local increase in calcium concentration caused by an action potential could loosen the bridge filament connections (Llinás et al., 1985; Chi et al., 2001) surrounding cluster filaments along the active zone. During vesicle fusion, the mechanical force of the vesicle collapsing into the membrane could pull an attached cluster filament, along with its complement of vesicles, closer to the active zone. A vesicle attached to that cluster filament could then become docked as it acquires multiple docking filaments, again nudging attached filaments closer to the active zone. Presumably, disused portions of the cluster filament are disassembled and depart from the active zone, as husks of cluster filaments are not found along the active zone membrane. This hypothetical sequence can explain why vesicles form stable clusters near active zones when recovering from intense cycles of vesicle release (Heuser et al., 1971) and why the dissociation of synapsin is vital for synaptic function (Llinás et al., 1985).

\section{References}

Allen C, Stevens CF (1994) An evaluation of causes for unreliability of synaptic transmission. Proc Natl Acad Sci U S A 91:10380-10383. CrossRef Medline

Baumeister W, Grimm R, Walz J (1999) Electron tomography of molecules and cells. Trends Cell Biol 9:81-85. CrossRef Medline

Burette AC, Lesperance T, Crum J, Martone M, Volkmann N, Ellisman MH, Weinberg RJ (2012) Electron tomographic analysis of synaptic ultrastructure. J Comp Neurol 520:2697-2711. CrossRef Medline

Chen X, Winters C, Azzam R, Li X, Galbraith JA, Leapman RD, Reese TS (2008a) Organization of the core structure of the postsynaptic density. Proc Natl Acad Sci U S A 105:4453-4458. CrossRef Medline

Chen X, Winters CA, Reese TS (2008b) Life inside a thin section: tomography. J Neurosci 28:9321-9327. CrossRef Medline

Chen X, Nelson CD, Li X, Winters CA, Azzam R, Sousa AA, Leapman RD, Gainer H, Sheng M, Reese TS (2011) PSD-95 is required to sustain the molecular organization of the postsynaptic density. J Neurosci 31:63296338. CrossRef Medline

Chen X, Winters C, Azzam R, Sousa AA, Leapman RD, Reese TS (2014) Nanoscale imaging of protein molecules at the postsynaptic density. In: Nanoscale imaging of synapses: new concepts and opportunities (Nägerl V, Triller A, eds), pp 1-21. New York: Humana.

Chi P, Greengard P, Ryan TA (2001) Synapsin dispersion and reclustering during synaptic activity. Nat Neurosci 4:1187-1193. CrossRef Medline

Denker A, Bethani I, Kröhnert K, Körber C, Horstmann H, Wilhelm BG, Barysch SV, Kuner T, Neher E, Rizzoli SO (2011) A small pool of vesicles maintains synaptic activity in vivo. Proc Natl Acad Sci U S A 108:1717717182. CrossRef Medline

Dubochet J (1995) High-pressure freezing for cryoelectron microscopy. Trends Cell Biol 5:366-368. CrossRef Medline

Fernández-Busnadiego R, Zuber B, Maurer UE, Cyrklaff M, Baumeister W, Lucic V (2010) Quantitative analysis of the native presynaptic cytomatrix by cryoelectron tomography. J Cell Biol 188:145-156. CrossRef Medline

Fowler MW, Staras K (2015) Synaptic vesicle pools: principles, properties and limitations. Exp Cell Res 335:150-156. CrossRef Medline

Frank J, Zhu J, Penczek P, Li Y, Srivastava S, Verschoor A, Radermacher M, Grassucci R, Lata RK, Agrawal RK (1995) A model of protein synthesis based on cryo-electron microscopy of the E. coli ribosome. Nature 376: 441-444. CrossRef Medline

Gitler D, Takagishi Y, Feng J, Ren Y, Rodriguiz RM, Wetsel WC, Greengard P, Augustine GJ (2004) Different presynaptic roles of synapsins at excitatory and inhibitory synapses. J Neurosci 24:11368-11380. CrossRef Medline

Gotow T, Miyaguchi K, Hashimoto PH (1991) Cytoplasmic architecture of the axon terminal: filamentous strands specifically associated with synaptic vesicles. Neuroscience 40:587-598. CrossRef Medline

Gustafsson JS, Birinyi A, Crum J, Ellisman M, Brodin L, Shupliakov O (2002) Ultrastructural organization of lamprey reticulospinal synapses in three dimensions. J Comp Neurol 450:167-182. CrossRef Medline

Harlow ML, Ress D, Stoschek A, Marshall RM, McMahan UJ (2001) The architecture of active zone material at the frog's neuromuscular junction. Nature 409:479-484. CrossRef Medline

Heuser J, Katz B, Miledi R (1971) Structural and functional changes of frog neuromuscular junctions in high calcium solutions. Proc R Soc Lond B Biol Sci 178:407-415. CrossRef Medline

Hirokawa N, Sobue K, Kanda K, Harada A, Yorifuji H (1989) The cytoskeletal architecture of the presynaptic terminal and molecular structure of synapsin 1. J Cell Biol 108:111-126. CrossRef Medline

Imig C, Min SW, Krinner S, Arancillo M, Rosenmund C, Südhof TC, Rhee J, Brose N, Cooper BH (2014) The morphological and molecular nature of synaptic vesicle priming at presynaptic active zones. Neuron 84:416-431. CrossRef Medline

Kremer JR, Mastronarde DN, McIntosh JR (1996) Computer visualization of three-dimensional image data using IMOD. J Struct Biol 116:71-76. CrossRef Medline

Landis DM, Hall AK, Weinstein LA, Reese TS (1988) The organization of cytoplasm at the presynaptic active zone of a central nervous-system synapse. Neuron 1:201-209. CrossRef Medline

Li F, Pincet F, Perez E, Eng WS, Melia TJ, Rothman JE, Tareste D (2007) Energetics and dynamics of SNAREpin folding across lipid bilayers. Nat Struct Mol Biol 14:890-896. CrossRef Medline

Llinás R, McGuinness TL, Leonard CS, Sugimori M, Greengard P (1985) Intraterminal injection of synapsin I or calcium/calmodulin-dependent protein kinase II alters neurotransmitter release at the squid giant synapse. Proc Natl Acad Sci U S A 82:3035-3039. CrossRef Medline

Mayer ML, Vyklicky L Jr (1989) The action of zinc on synaptic transmission and neuronal excitability in cultures of mouse hippocampus. J Physiol 415:351-365. CrossRef Medline

McIntosh JR (2001) Electron microscopy of cells: a new beginning for a new century. J Cell Biol 153:F25-F32. CrossRef Medline

Moore H (1987) Theory and practice of high pressure freezing. In: Cryotechniques in biological electron microscopy (Steinbrecht RA, Zierold K, eds), pp 175-191. Berlin: Springer.

Murthy VN, Sejnowski TJ, Stevens CF (1997) Heterogeneous release properties of visualized individual hippocampal synapses. Neuron 18:599612. CrossRef Medline

Rickman C, Hu K, Carroll J, Davletov B (2005) Self-assembly of SNARE fusion proteins into star-shaped oligomers. Biochem J 388:75-79. CrossRef Medline

Schikorski T, Stevens CF (1997) Quantitative ultrastructural analysis of hippocampal excitatory synapses. J Neurosci 17:5858-5867. Medline

Siksou L, Rostaing P, Lechaire JP, Boudier T, Ohtsuka T, Fejtová A, Kao HT, Greengard P, Gundelfinger ED, Triller A, Marty S (2007) Threedimensional architecture of presynaptic terminal cytomatrix. J Neurosci 27:6868-6877. CrossRef Medline

Siksou L, Triller A, Marty S (2009) An emerging view of presynaptic structure from electron microscopic studies. J Neurochem 108:1336-1342. CrossRef Medline

Siksou L, Triller A, Marty S (2011) Ultrastructural organization of presynaptic terminals. Curr Opin Neurobiol 21:261-268. CrossRef Medline

Smith JE, Reese TS (1980) Use of aldehyde fixatives to determine the rate of synaptic transmitter release. J Exp Biol 89:19-29. Medline

Stevens CF, Wang Y (1994) Changes in reliability of synaptic function as a mechanism for plasticity. Nature 371:704-707. CrossRef Medline

Stigloher C, Zhan H, Zhen M, Richmond J, Bessereau JL (2011) The presynaptic dense projection of the Caenorhabditis elegans cholinergic neuromuscular junction localizes synaptic vesicles at the active zone through SYD-2/liprin and UNC-10/RIM-dependent interactions. J Neurosci 31: 4388-4396. CrossRef Medline

Südhof TC, Rothman JE (2009) Membrane fusion: grappling with SNARE and SM proteins. Science 323:474-477. CrossRef Medline

Szule JA, Harlow ML, Jung JH, De-Miguel FF, Marshall RM, McMahan UJ (2012) Regulation of synaptic vesicle docking by different classes of macromolecules in active zone material. PLoS One 7:e33333. CrossRef Medline

Takamori S, Holt M, Stenius K, Lemke EA, Grønborg M, Riedel D, Urlaub H, Schenck S, Brügger B, Ringler P, Müller SA, Rammner B, Gräter F, Hub JS, De Groot BL, Mieskes G, Moriyama Y, Klingauf J, Grubmüller H, Heuser J, Wieland F, Jahn R (2006) Molecular anatomy of a trafficking organelle. Cell 127:831-846. CrossRef Medline

Watanabe S, Rost BR, Camacho-Pérez M, Davis MW, Söhl-Kielczynski B, Rosenmund C, Jorgensen EM (2013) Ultrafast endocytosis at mouse hippocampal synapses. Nature 504:242-247. CrossRef Medline

Zampighi GA, Zampighi LM, Fain N, Lanzavecchia S, Simon SA, Wright EM (2006) Conical electron tomography of a chemical synapse: vesicles docked to the active zone are hemi-fused. Biophys J 91:2910-2918. CrossRef Medline 